\title{
Bar coding vaccines - More than a 'check-out' issue
}

\author{
Monika Naus MD FRCPC FACPM \\ Member, National Advisory Committee on Immunization
}

$\mathrm{I}_{\mathrm{i}}$ have a dream. That dream is that a technology in wide use in the private and retail sectors could change the way that we do business in our immunization programs. Immunization is a simple and routine procedure for the majority of Canadian children, and provides a large benefit of protection. Most provincial immunization programs currently provide vaccines against diphtheria, tetanus, pertussis, polio, Haemophilus influenzae type b, measles, mumps, rubella and hepatitis B. In my province, Ontario, these vaccines are given to a child or adolescent during 10 clinical encounters, six during the preschool period and four during adolescence. In the coming years, we expect further additions to the vaccine list, including varicella and pneumococcal disease, and perhaps influenza and hepatitis A. In the more distant future, a myriad of other diseases may be added to the 'vaccine preventable' list, including meningococcal disease and sexually transmitted diseases. Vaccination programs for adults are also receiving attention in recent years and, over time, may be incorporated into expansions of 'childhood' immunization registries. As the complexity of immunization schedules grows, we need to look at simplifying our means of recording, providing, collecting and sharing this information for the benefit of the individual and the population.

One such tool that could simplify our procedures is the use of bar codes on vaccine packages. Much to my surprise, this is not a technology that has entered routine use in the vaccine manufacturing sector. Apart from the neighbourhood 'mom and pop' grocery store, bar code technology is in widespread use in the retail sector. It saves time for the check-out staff, reduces errors inherent in manual data entry and provides 'realtime' inventory information to management. It provides informative verification to me, the customer, about which items I purchased and how much I paid.

No such technology is in use in our hugely important, large transactional public immunization programs. These programs distribute an estimated $\$ 100$ million worth of vaccines each year in Canada. The Canadian Immunization Guide, 5th edition (1) recommends that health care providers who administer vaccines record the following items on the patient's medical chart: trade name of the product; date given; dose; site and route of administration; manufacturer; and lot number. Most of the time, the data recorded are limited to the common abbreviation for the vaccine (eg, MMR for measles, mumps and rubella vaccines) and date of administration. In the few instances where an investigation is needed because of a subsequent untoward adverse vaccine event, the lot number of the vaccine given is not known and the product identification is surmised based on which products were in use at that time in the jurisdiction. This is surely 'circumstantial' evidence at best. Because of the many items needed to establish a product's identity, these may not be recorded at each step of the distribution system. As a result, the system's ability to carry out product recalls down to the end user is limited. Thanks to the public funding of vaccine programs, most, if not all, Canadian jurisdictions have a central pharmacy warehouse and a larger number of regional depots for vacinne distribution. This situation ideally lends itself to an automated inventory management system for product reordering and per capita utilization assessment. Instead, most inventory systems beyond the central warehouse are paper-based.

Several initiatives are under way to develop standardized data elements for existing or developing immunization registries in the provinces and territories. Health Canada is coordinating these initiatives in response to recommendations issued by the Canadian Consensus Conference on a National Immunization Records System, held in March 1998. The 'smart card' initiatives in many provinces and territories, which embed key minimal data sets on an individual's health card, lend themselves perfectly to the maintenance of immunization records. Now, if we could only make these records accurate by using bar code technology!

Now is the time for vaccine manufacturers to incorporate bar codes on immunizing agents. Perhaps this should be a component of the labelling required by the Bureau of Biologics and Radiopharmaceuticals of Health Canada. The use of bar coding would increase the speed, accuracy and completeness of recording information about immunizing agents. Combined with other innovations of our record keeping and data sharing needs for public immunization programs, it would benefit:

- the individual by providing an accurate record of their immunization history; 
- the health care provider who is trying to keep an accurate record of vaccines given;

- the public health sector when trying to assess immunization rates and ensure product safety; and

- the vaccine inventory management staff in provincial and local depots.

A standard format for bar coding is currently being discussed with manufacturers by the United States Centers for Disease Control and Prevention, Atlanta, Georgia. Technology for reading bar codes is inexpensive, and exists or could soon exist in vaccine distribution depots, including the manufacturers, provincial or territorial government pharmacies, and regional or local public health departments. With the increased use of computer technology in physicians' offices for patient and office management, bar code technology can reasonably come into use in these settings in the coming decade.

\section{REFERENCES}

1. National Advisory Committee on Immunization. Canadian Immunization Guide, 5th edn. Ottawa: Supply and Services Canada, 1998.

\section{BOOK REVIEWS}

\section{Viral Hepatitis: Diagnosis, Therapy, and Prevention (1999). Steven Spector, ed. Humana Press Inc, Totowa, New Jersey. ISBN 0-89603-424-0; 402 pages; US\$99.50}

Viral hepatitis is a rapidly expanding area, in which new hepatotrophic viruses are being discovered and our understanding, treatment and ability to prevent infections with these agents continue to improve. Thus, new texts that address these important developments are always welcome. Viral Hepatitis, Diagnosis, Therapy and Prevention, a 402page, multi-authored effort, is no exception and, in many respects, is quite an improvement over similar texts published to date in this area. The majority of the 220 contributors are considered authorities in their respective fields, and their international make-up (from the United States, United Kingdom and Italy) brings a broad perspective to the epidemiology, and perhaps our understanding, of the natural history of these infections. The text is neatly divided into an introduction and 10 chapters. The first six chapters outline the biology, pathogenesis, epidemiology, clinical description and diagnosis of hepatitis A, B, C, D, E and the recently discovered $G B$ virus. The chapters dealing with hepatitis D, E and GB also contain sections on therapy, prevention and discovery. One chapter is devoted to laboratory diagnoses of all forms of viral hepatitis, and one chapter specifically addresses antiviral therapy for those viruses that cause chronic infections. The final two chapters deal with vaccine developments for hepatitis A and hepatitis B (HBV) viruses.

The text has a number of attractive features. For the most part, the writing is clear and concise. Overlap, despite the multi-authored format and chapter development, has been kept to a minimum. Another positive feature is the depth with which the majority of topics are dealt. This is particularly evident in the sections dealing with the viruses themselves and their replicative cycles. For example, interested readers will be pleased to find descriptions of the hairpin structures that might participate in regulation of hepatitis $C$ virus genome expression and the precise positions of large stem loops, which are thought to represent internal ribosomal entry sites.

In terms of the book's problems, the detail given to virology appears to have come at the expense of other features of the infections in some of the chapters. For example, while extensively discussing the structure of hepatitis $\mathrm{C}$ virus and its gene products, the treatment chapter limits discussion of present therapy (interferon and ribavirin) to two short paragraphs. This oversight cannot be ascribed to the relatively recent emergence of combination therapy because 1998 references are found in numerous areas elsewhere in the text. Other examples of important topics given short shrift are found in the HBV vaccine chapter where there is no mention of who is unlikely to respond to $\mathrm{HBV}$ vaccine and the reasons for the lack of response. The answer to whether routine testing for anti-HBs should be obtained postvaccination in all recipients or only in certain subgroups (and, if the latter, in which subgroups) is also not to be found. Less disconcerting but still irritating are occasional typographical errors and references to pages in the index that are either incorrect or do not exist. Finally, the quality of some of the figures is limited as are the numbers of figures in certain chapters, such as the hepatitis D and HBV chapters.

In summary, this textbook deals with an important and timely subject. For the virologist, the answers to many if not all questions dealing with hepatotrophic viruses can be found within its pages. However, those virologists and clinicians (hepatologists, infectious disease specialists, gastroenterologists, etc) wanting to know similar detail about the clinical features of viral hepatitis will be disappointed. Fortunately, all interested parties will usually find adequate and up-to-date references to complement the brevity of certain sections.

Who should have this text on their bookshelves? In this reviewer's opinion, it is a recommended purchase for virologists, hepatologists and infectious disease specialists. Its relevance and usefulness to gasteroenterologists, internists and family practitioners is limited.

Gerald Minuk MD FRCPC Health Sciences Centre Winnipeg, Manitoba 


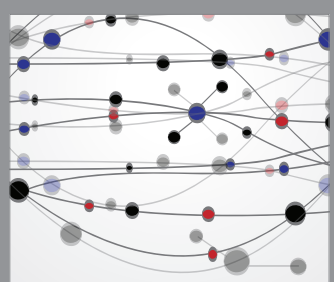

The Scientific World Journal
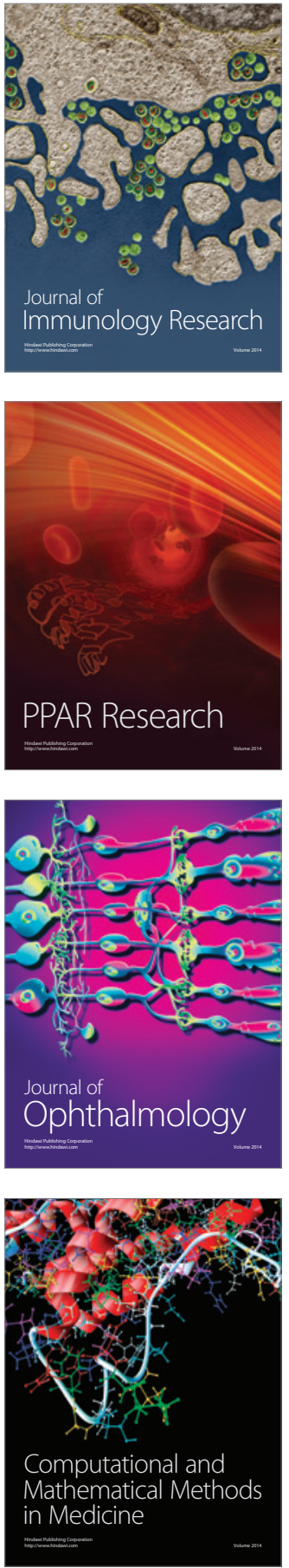

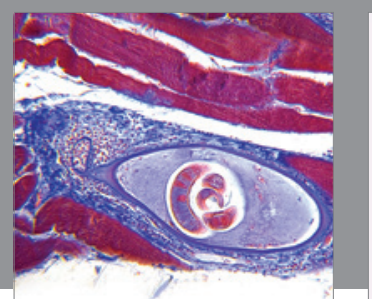

Gastroenterology Research and Practice

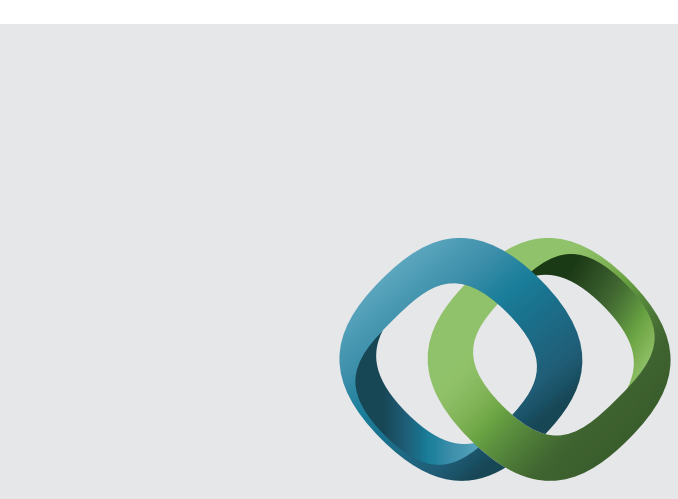

\section{Hindawi}

Submit your manuscripts at

http://www.hindawi.com
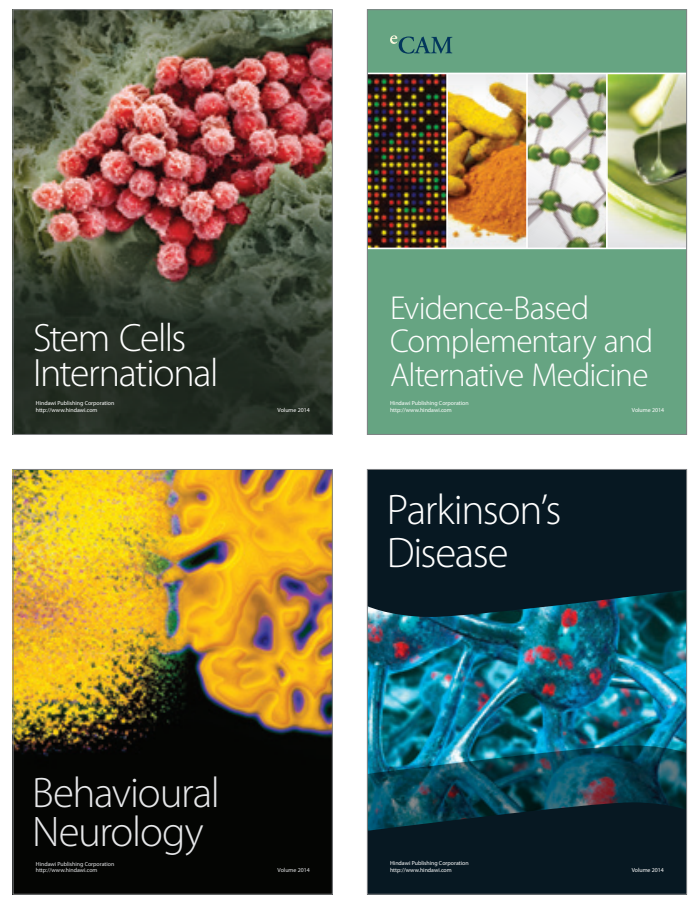
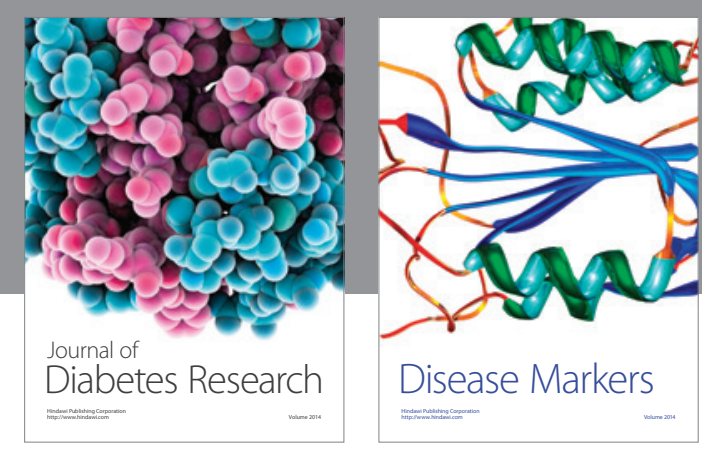

Disease Markers
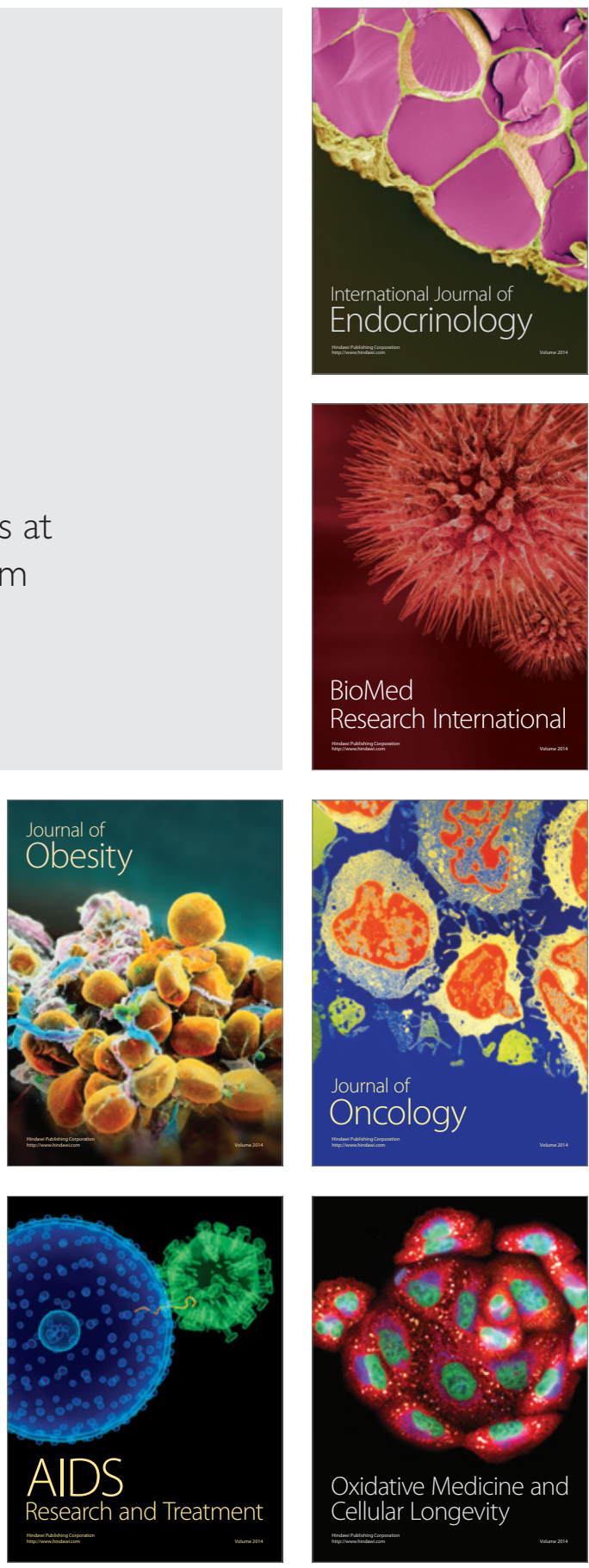